\title{
Hepatic expression of the Sptlc3 subunit of serine palmitoyltransferase is associated with the development of hepatocellular carcinoma in a mouse model of nonalcoholic steatohepatitis
}

\author{
YOZO YOSHIMINE $^{1}$, HIROFUMI UTO $^{1}$, KOTARO KUMAGAI $^{1}$, SEIICHI MAWATARI $^{1}$, SHIHO ARIMA $^{1}$, \\ RIE IBUSUKI $^{1}$, KUMIKO MERA ${ }^{1}$, TSUYOSHI NOSAKI ${ }^{1}$, SHUJI KANMURA ${ }^{1}$, MASATSUGU NUMATA ${ }^{1}$, \\ TSUTOMU TAMAI $^{1}$, AKIHIRO MORIUCHI ${ }^{2}$, HIROHITO TSUBOUCHI ${ }^{2,3}$ and AKIO IDO ${ }^{1,2}$ \\ ${ }^{1}$ Digestive and Lifestyle Diseases, Department of Human and Environmental Sciences; \\ ${ }^{2}$ Department of HGF Tissue Repair and Regenerative Medicine, Kagoshima University \\ Graduate School of Medical and Dental Sciences; ${ }^{3}$ Kagoshima City Hospital, Kagoshima, Japan
}

Received September 29, 2014; Accepted December 1, 2014

DOI: $10.3892 /$ or.2015.3745

\begin{abstract}
The molecular mechanisms underlying the progression of nonalcoholic steatohepatitis (NASH) have not been fully elucidated. The aim of this study was to identify factors involved in NASH progression by analysis of pathophysiological features and gene-expression profiles in livers of STAM mice, a model of NASH-associated hepatocarcinogenesis. C57BL/6N (B6N) mice were injected with streptozotocin to generate STAM mice. Four-week-old male STAM and B6N mice were fed a high-fat diet (HFD) (STAM-F, B6N-F) or a conventional diet (STAM-C, B6N-C) until they were 10, 14, or 18 weeks old. Blood glucose and nonalcoholic fatty liver disease (NAFLD) activity scores of STAM-F were higher than those of STAM-C during all observation periods. STAM-F mice had more severe hepatic fibrosis at 14 weeks, and exhibited higher levels of $\alpha$-fetoprotein-positive hepatic tumor formation with multiplication than STAM-C mice at 18 weeks. At 14 weeks, cDNA microarray analysis revealed that the hepatic expression of eight mRNAs was $\geq 30$-fold higher in STAM-F than B6N-F mice. The expression of another four genes was increased $\geq 5$-fold in STAM-F than B6N-F mice, and $\geq 5$-fold in $\mathrm{B} 6 \mathrm{~N}-\mathrm{F}$ relative to $\mathrm{B} 6 \mathrm{~N}-\mathrm{C}$ mice. Of the 12 genes, the difference in Sptlc3 mRNA expression was most pronounced, and gradually increased over time, as determined by quantitative RT-PCR in STAM-F mice. In addition, Sptlc3 mRNA expression in STAM-F mice was higher than that in $\mathrm{db} / \mathrm{db}$ mice
\end{abstract}

Correspondence to: Dr Hirofumi Uto, Digestive and Lifestyle Diseases, Department of Human and Environmental Sciences, Kagoshima University Graduate School of Medical and Dental Sciences, 8-35-1 Sakuragaoka, Kagoshima 890-8520, Japan

E-mail: hirouto@m2.kufm.kagoshima-u.ac.jp

Key words: Sptlc3, Mmp13, Grip1, Cxcl14, nonalcoholic steatohepatitis, insulin secretion that received HFD and in $\mathrm{B} 6 \mathrm{~N}$ mice fed a choline-deficient L-amino acid (CDAA)-defined diet. In conclusion, a high-fat diet aggravated pathophysiological findings in the liver in NASH mouse models, and the hepatic expression of Sptlc3 mRNA was potentially associated with NASH progression.

\section{Introduction}

Nonalcoholic fatty liver disease (NAFLD) is a spectrum of liver conditions with histopathological features similar to alcoholic liver disease that arise in patients with no apparent history of alcohol intake. NAFLD is classified into two main types: nonalcoholic fatty liver (NAFL), characterized simply by deposition of fat in the liver, and nonalcoholic steatohepatitis (NASH), characterized by hepatic inflammation with hepatocyte injury (ballooning), with or without fibrosis, as well as hepatic steatosis (1). NAFL and NASH are considered to be hepatic manifestations of metabolic syndrome, because the presence of obesity and obesity-induced insulin resistance is fundamentally associated with the pathogenesis and progression of the two diseases. Consequently, NAFL and NASH are often accompanied by complications such as diabetes, hypertension, and dyslipidemia (2). However, the molecular mechanisms underlying the progression of NAFL and NASH in association with metabolic syndrome have not yet been fully elucidated.

NASH can lead from progressive hepatic disease to liver cirrhosis (LC), with subsequent development of hepatocellular carcinoma (HCC) (3). The incidence rate of NASH-derived $\mathrm{HCC}$ without complication by LC was found to range from 0 to $3 \%$ over a 20 -year period, whereas the rate of NASH-derived HCC with LC was higher, ranging from $2.4 \%$ over a 7 -year period to $12.8 \%$ over a 3 -year period (4). Although the incidence of $\mathrm{HCC}$ is lower in patients with NASH than in patients with hepatitis $\mathrm{C}$ virus (HCV) infection, the number of patients with NASH-associated HCC is expected to increase along with the growing incidence of metabolic syndrome $(4,5)$. 
According to the two-hit theory, a widely known hypothesis aimed at explaining the pathogenesis and progression of NAFLD/NASH, onset of hepatic fat deposition is the "first hit', while the 'second hit' can be due to any of several factors, including hepatocellular injury and genetic predisposition, and leads to the onset of NASH (6). Although a large part of the disease process remains unexplained by the two-hit theory, it is now widely believed that many of the risk factors are intricately associated with each other. Dietary and/or genetic factors, along with inflammatory mediators of intestinal origin or those derived from fat tissue, may contribute in parallel to the development of NASH. Tilg et al have characterized this process by introducing the concept of 'multiple parallel hits' (7).

Since factors that contribute to the pathogenesis of NASH leading to LC and HCC remain unclear, we investigated the pathological processes of NASH-associated hepatocarcinogenesis using animal models. No fibrosis formation or hepatocarcinogenesis have been reported in studies with conventional models using leptin-deficient ob/ob mice, or in high-fat or high-fructose diet models (8), even though hyperglycemia, insulin resistance, and obesity were observed under these conditions. In models that use mice fed a choline-deficient L-amino acid (CDAA)-defined diet, no abnormal glucose tolerance or obesity has been clearly observed, despite the development of steatosis and subsequent hepatic fibrosis, the latter of which is ultimately associated with LC and hepatocarcinogenesis (9). Thus, no studies have accurately recapitulated the pathogenesis of human NASH in conventional animal models. In a model using STAM mice fed a high-fat diet after the onset of pancreatic dysfunction induced by streptozotocin administration, findings of a recent study showed the development of steatosis, hepatic fibrosis, and HCC over shorter periods (10). The STAM mouse model has drawn attention because the animals predictably and consistently develop pathologies similar to human NASH, although hyperinsulinemia is not observed in this mice model. However, the molecular mechanisms of hepatocarcinogenesis in this model have not been fully investigated. In this study, we investigated whether a high-fat diet affects hepatocarcinogenesis in a NASH model induced by hyperglycemia, and explored the genetic associations with the pathogenic mechanisms of hepatocarcinogenesis.

\section{Materials and methods}

Animals. To generate STAM mice, two-day-old male C57BL/6N (B6N) mice received subcutaneous injection of $200 \mu \mathrm{g}$ streptozotocin to reduce pancreatic function. Male STAM mice were purchased from Stelic Institute \& Co. (Tokyo, Japan). Starting at four weeks of age, STAM mice were fed a high-fat diet (HFD) (STAM-F) or conventional diet (STAM-C). Four-week-old male B6N mice were purchased from Charles River Laboratories Japan, Inc., (Yokohama, Japan). Similarly, starting at four weeks of age, B6N mice were fed a high-fat diet (B6N-F) or conventional diet (B6N-C). In the high-fat diet, $60 \%$ of the calories (HFD-60) were derived from fat (Oriental Yeast Co., Ltd., Tokyo, Japan), while the conventional diet (CE-2) contained $4.6 \%$ fat (CLEA Japan, Inc., Tokyo, Japan). The mice were kept in standard wire cages and allowed free access to food and water. The animals were then weighed, and blood pressure (Model MK-1030, Blood Pressure Monitor For Rats \& Mice, Muromachi Kikai Co., Ltd., Tokyo, Japan) and serum fasting blood glucose (FBG) were measured weekly. The animals were sacrificed at 10,14 , or 18 weeks. Subsequently, the mice were fasted for $12 \mathrm{~h}$ prior to sacrifice, and serum and liver samples were collected and stored at $-80^{\circ} \mathrm{C}$ prior to use.

Male C57BLKS/J lar- $+\operatorname{Lepr}^{\mathrm{db}} /+\mathrm{Lepr}^{\mathrm{db}}(\mathrm{db} / \mathrm{db})$ mice were purchased from Japan SLC, Inc. (Hamamatsu, Japan). These mice were established as a type 2 diabetes mouse model. Five-week-old $\mathrm{db} / \mathrm{db}$ mice were fed an HFD and sacrificed at 25 weeks (db/db-F group). In addition, four-week-old B6N mice were fed a CDAA diet (Dyets, Bethlehem, PA, USA) and sacrificed at 16 weeks (CDAA group).

The animal experiments were approved by the Institutional Animal Care and Use Committee guideline of Kagoshima University Graduate School of Medical and Dental Sciences.

Biochemical examination. Aspartate aminotransferase (AST) and alanine aminotransferase (ALT) levels in mice were measured using Spotchem ${ }^{\mathrm{TM}}$ EZ SP-4430 (Arkray, Inc., Kyoto, Japan). Fasting blood glucose (FBG) levels were measured using Cyclic GB sensor (Eidia Co., Ltd., Tokyo, Japan). Serum insulin levels were measured using an enzyme-linked immunosorbent assay (ELISA, Morinaga Institute of Biological Science, Inc., Yokohama, Japan) according to the manufacturer's instructions.

Histological examination. Liver tissues were isolated and fixed in $10 \%$ neutralized formalin buffer solution. The samples were then embedded in paraffin and sliced at a thickness of 4-5 $\mu \mathrm{m}$. Liver pathology was evaluated by staining with hematoxylin/eosin.

Accumulation of fat in hepatocytes was evaluated by oil red O staining. Liver tissue section were covered with O.C.T gel and stored at $-80^{\circ} \mathrm{C}$. Frozen sections of liver tissue $(<6 \mu \mathrm{m})$ were fixed in $10 \%$ neutralized formalin buffer solution for 30 min and then rinsed with 60\% isopropanol. After washing with distilled water, the nuclei were stained with Mayer's hematoxylin for $1 \mathrm{~min}$, and then rinsed again with distilled water. Quick Grain Standard (Inotech Co., Ltd, Hiroshima, Japan) was used to measure the area positive for oil red O staining.

Liver fibrosis was evaluated by Sirius red staining. Sections $(4 \mu \mathrm{m})$ were fixed in saturated picric acid and $1 \%$ Sirius red for $90 \mathrm{~min}$ at room temperature. Positively stained areas were measured using Quick Grain Standard.

Activity of NAFL/NASH was assessed using the NAFLD Activity Score (NAS), as described by Kleiner et al (11), a composite parameter based on separate scores for steatosis (0-3), lobular inflammation (0-3), and hepatocellular ballooning (0-2). The NAS is the sum of these scores: values $\geq 5$ are correlated with a diagnosis of NASH in humans (11).

Immunohistochemistry. Hepatic tumors were assessed by immunostaining for placental glutathioneS-transferase(GST-P) and heat shock protein (HSP)-70. A rabbit polyclonal antibody against GST-P (Medical \& Biological laboratories Co., Ltd, Nagoya, Japan) was used at a dilution of 1:2,000, and a rabbit polyclonal antibody against HSP-70 (Cell Signaling Technology, Inc.) was used at a dilution of 1:25. 
Table I. Oligonucleotide sequence of primers for quantitative RT-PCR.

\begin{tabular}{lll}
\hline Genes & \multicolumn{1}{c}{ Forward primer $\left(5^{\prime}-3^{\prime}\right)$} & \multicolumn{1}{c}{ Reverse primer (5'-3') } \\
\hline Sptlc3 & TTTGGACTGGACCCTGAAGATATTG & TGACTGCATCCGTAAATAATCCACA \\
Gripl 1 & TCCCTGGAATTGGCAACAAAG & GGAATTTGTTGGCATGACTCTCAC \\
Cxcl14 & TGAAGCCCTACGACAGGCTCTTA & GTCCAGCTTGTTGCCAGATTCA \\
Afp & CAAAGTACCCACACTGCGAGGA & CCAGGCATTGTACCACTTGATGA \\
Gpc3 & AGTGCTGCAAAGCCCAGGA & TGGAGATGTTTAAACGCCCAAAG \\
Actb & ACTGCGGTGATGATGAAGATGAA & GCATCGTCCACATCCAGATCATA \\
\hline
\end{tabular}

Gene expression profile. Liver tissues of 14-week-old B6N-C, B6N-F and STAM-F mice were homogenized in TRIzol solution (Life Technologies, Carlsbad, CA, USA), and RNA was then isolated according to the manufacturer's instructions. En bloc total RNA from five mice was subjected to expression profiling on an Affymetrix GeneChip Mouse genome 430 2.0 array according to a standard protocol (Affimetrix, Santa Clara, CA, USA). The resultant expression profiles were normalized using the GeneSpring GX 11 software (Agilent Technologies, Santa Clara, CA, USA).

Quantitative RT-PCR. Template cDNA was synthesized from $0.5 \mu \mathrm{g}$ of total RNA using a PrimeScript RT reagent kit (Takara Bio Inc., Otsu, Japan). Following microarray analysis, we identified genes that were expressed more highly in STAM-F than in B6N-F and B6N-C. The expression levels were subsequently detected in liver tissue by quantitative RT-PCR using SYBR Premix Ex Taq ${ }^{\mathrm{TM}}$ II (Takara Bio Inc.) on a StepOnePlus ${ }^{\mathrm{TM}}$ real-time PCR system. The level of each mRNA was normalized to that of $\beta$-actin (Actb) mRNA in the same sample. The following mRNAs were examined in this manner: serine palmitoyltransferase long chain base subunit 3 (Sptlc3), matrix metallopeptidase 13 (Mmp13), glutamate receptor interacting protein 1 (Gripl), chemokine (C-X-C motif) ligand 14 (Cxcl14), $\alpha$-fetoprotein (Afp), glypican $3(G p c 3)$, and $\beta$-actin $(A c t b)$. Primer sequences are provided in Table I.

Statistical analysis. Results are presented as means \pm standard deviations. Statistical evaluation was performed with IBM SPSS 15.0J (IBM Corporation, Armonk, NY, USA) for Windows. Data were analyzed for statistical differences using the Mann-Whitney U test or Tukey's HSD test. $\mathrm{P}<0.05$ was considered statistically significant.

\section{Results}

Physical findings in B6N and STAM mice. Body weight gradually increased in B6N-F mice, but no increase was observed in either of the STAM groups between 4 and 18 weeks of age (Fig. 1A). The dietary calorie intake of STAM-F was 1.3-fold higher that of STAM-C mice, although there was no significant difference in weight between the two groups. Blood pressure did not differ significantly during the observed periods: specifically, systolic blood pressure was $\sim 100 \mathrm{mmHg}$ in all groups each week.

Biochemical examination in STAM and B6N mice. Serum ALT levels decreased from 10 weeks of age ( 6 weeks of diet) to 18 weeks (14 weeks of diet) in B6N-C and STAM-C mice, but increased in B6N-F and STAM-F mice (Fig. 1B). In addition, ALT levels in the B6N-F groups were higher than those in the B6N-C groups at 10, 14 and 18 weeks, and ALT levels in STAM-F were significantly higher than those in STAM-C at 18 weeks (Fig. 1B).

The two STAM groups, in which pancreatic function was degraded, had high $\mathrm{FBG}$ relative to the $\mathrm{B} 6 \mathrm{~N}$ groups, and FBG was significantly higher in the STAM-F (>400 mg/dl) than in the STAM-C, B6N-F and B6N-C groups (Fig. 1C). By contrast, serum insulin levels were lower in the two STAM groups, and were significantly higher in B6N-F than in the other three groups (Fig. 1D).

Hepatic steatosis in STAM and B6N mice. There was no apparent hepatic steatosis in $\mathrm{B} 6 \mathrm{~N}-\mathrm{C}$ mice at 10,14 , or 18 weeks (Fig. 2), but hepatic steatosis in the B6N-F group gradually increased over time, as observed at 10, 14 and 18 weeks (Fig. 2 and 3B). By contrast, STAM-F mice at 10 weeks had apparent hepatic steatosis (Fig. 2 and 3A), and the area of hepatic steatosis in the STAM-F group was lower at 14 and 18 weeks than at 10 weeks (Fig. 3B). There was no apparent hepatic steatosis in STAM-C mice during the experimental period.

Hepatic fibrosis in STAM and B6N mice. Hepatic fibrosis, assessed by image analysis of Sirius red staining, was more severe in STAM-F mice at 10 (Fig. 4A) and 14 (Fig. 4B) weeks than in the B6N-C (Fig. 4D), B6N-F (Fig. 4E), and STAM-C mice (Fig. 4F) during the same period (Fig. 4G). By contrast, the area of hepatic fibrosis in STAM-F mice at 18 weeks (Fig. 4C) decreased relative to 10 or 14 weeks, and the degree of fibrosis was similar to those in the B6N-F and STAM-C groups (Fig. 4G).

NAFLD activity score (NAS) in STAM and B6N mice. The NAS, a composite parameter calculated from separate scores for steatosis, inflammation, and ballooning, was higher in B6N-F and the two STAM groups than in B6N-C. Between 


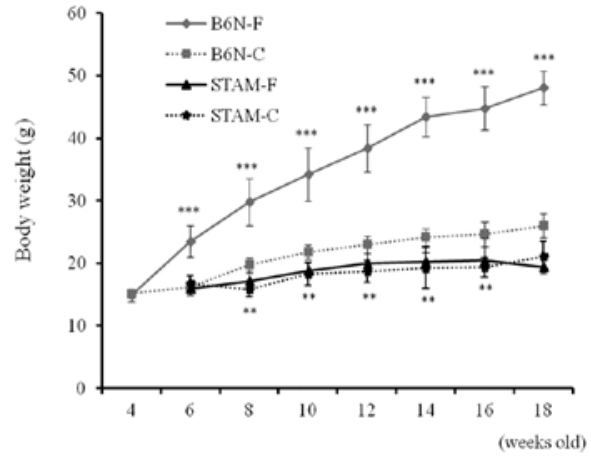

B

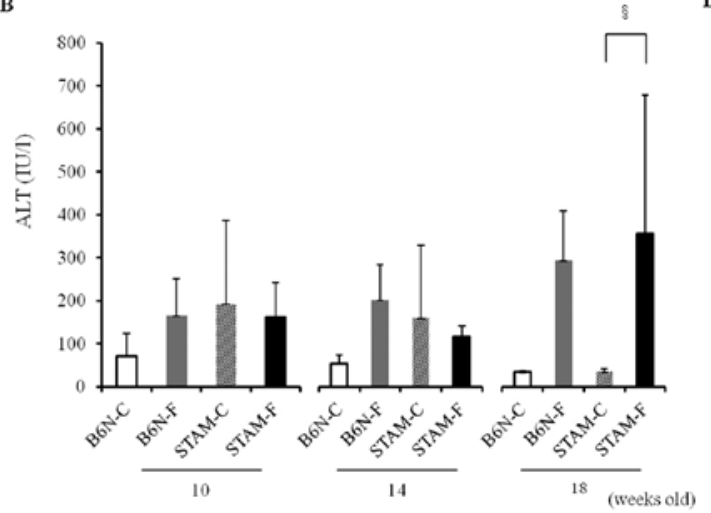

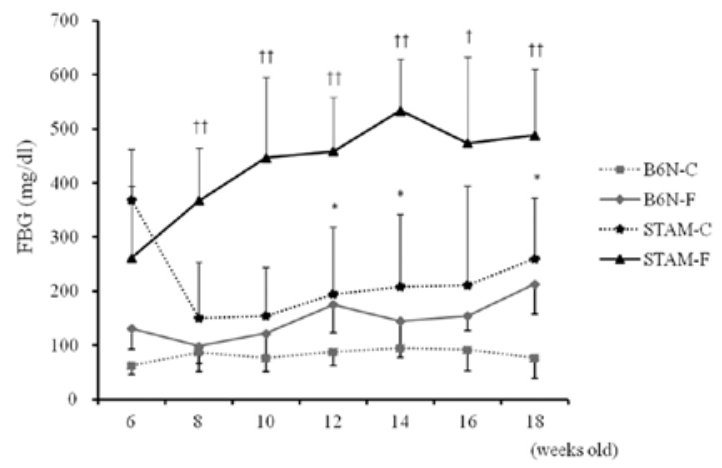

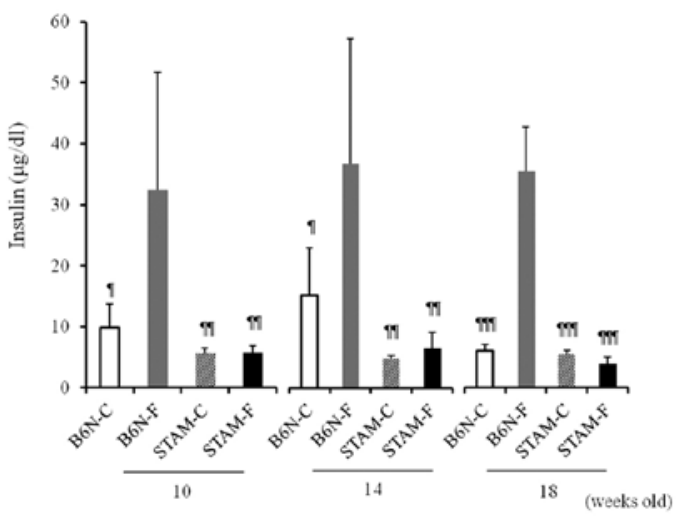

Figure 1. Body weights and serum markers in B6N and STAM mice. (A) Body weight. (B-D) Serum levels of (B) ALT, (C) fasting blood glucose and (D) insulin. Data are presented for each group as means \pm standard deviation. Results shown are for: (A and C) $n=15$ in each group at 4-10 weeks; $n=10$ in each group at 12-14 weeks and $n=5$ in each group at 16 and 18 weeks. (B and $D) n=5$ in each group at 10, 14, and 18 weeks. Statistical analyses were performed using Tukey's HSD test. ${ }^{*} \mathrm{p}<0.05,{ }^{* *} \mathrm{p}<0.01,{ }^{* * *} \mathrm{p}<0.001$ vs. B6N-C each week; ${ }^{\dagger} \mathrm{p}<0.05,{ }^{\prime \dagger} \mathrm{p}<0.001$ vs. B6N-F and STAM-C each week; ${ }^{9} \mathrm{p}<0.05,{ }^{\mathrm{II}} \mathrm{p}<0.01,{ }^{959} \mathrm{p}<0.001 \mathrm{vs}$. B6N-F each week; ${ }^{\circledR} \mathrm{p}<0.05$, STAM-C vs. STAM-F.

$\mathrm{B} 6 \mathrm{~N}-\mathrm{C}$

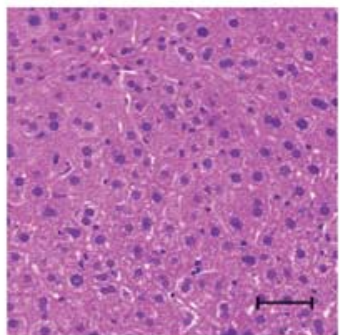

14 weeks

10 weeks

8 weeks
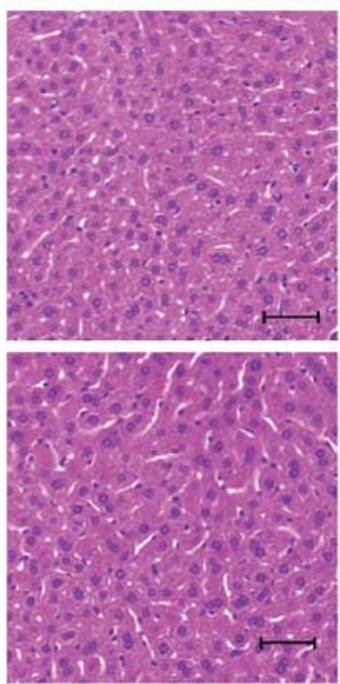

$\mathrm{B} 6 \mathrm{~N}-\mathrm{F}$
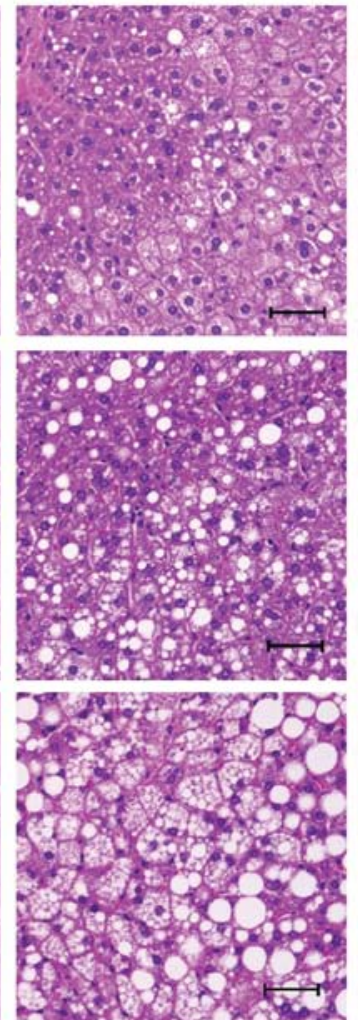

STAM-C
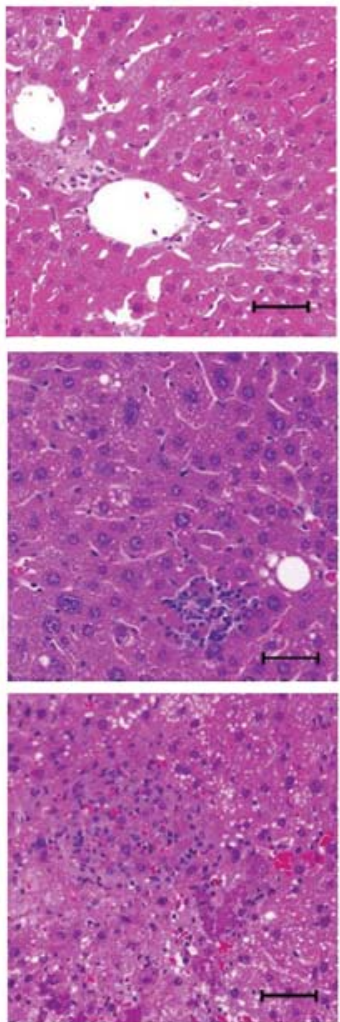

STAM-F
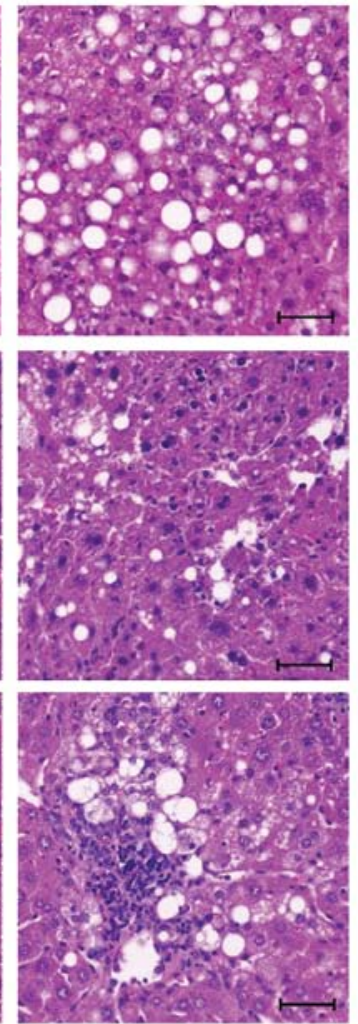

Figure 2. Histological features of the liver based on hematoxylin and eosin staining. Hematoxylin and eosin staining of the liver in B6N-C, B6N-F, STAM-C and STAN-F mice at 10, 14 and 18 weeks. Original magnifications, x200; scale bar, $50 \mu \mathrm{m}$. 


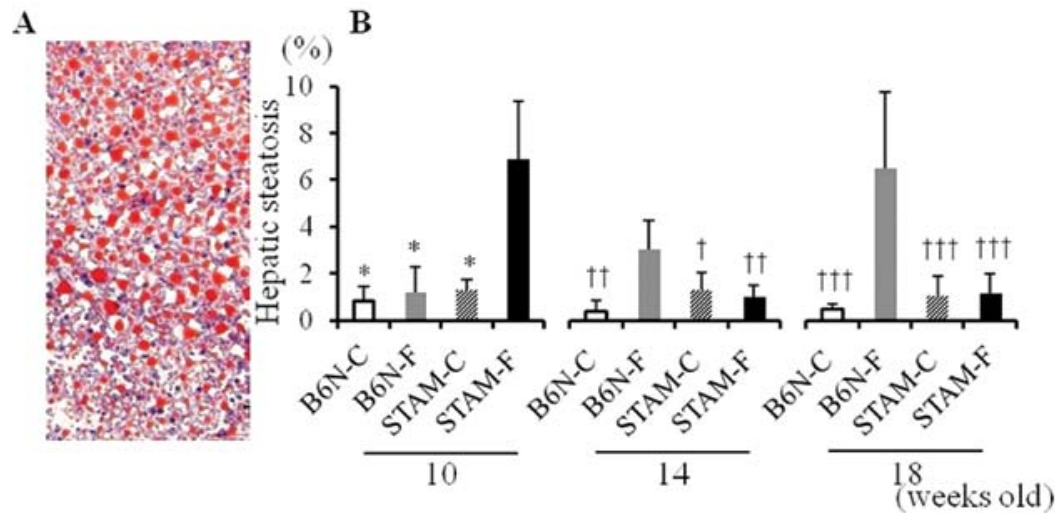

Figure 3. Hepatic steatosis based on oil red O staining. (A) Oil red O staining of the liver in STAM-F mice at 10 weeks. Original magnifications, x200; scale bar, $50 \mu \mathrm{m}$. (B) Area positive for oil red O staining in B6N and STAM mice at 10, 14 and 18 weeks ( $\mathrm{n}=5$ in each group). Statistical analyses were performed using Tukey's HSD test. At 10 weeks, ${ }^{*}$ p $<0.001$ vs. STAM-F. At 14 and 18 weeks, ${ }^{\dagger} \mathrm{p}<0.05,{ }^{\dagger \dagger} \mathrm{p}<0.01,{ }^{\dagger \dagger} \mathrm{p}<0.001$ vs. B6N-F mice.
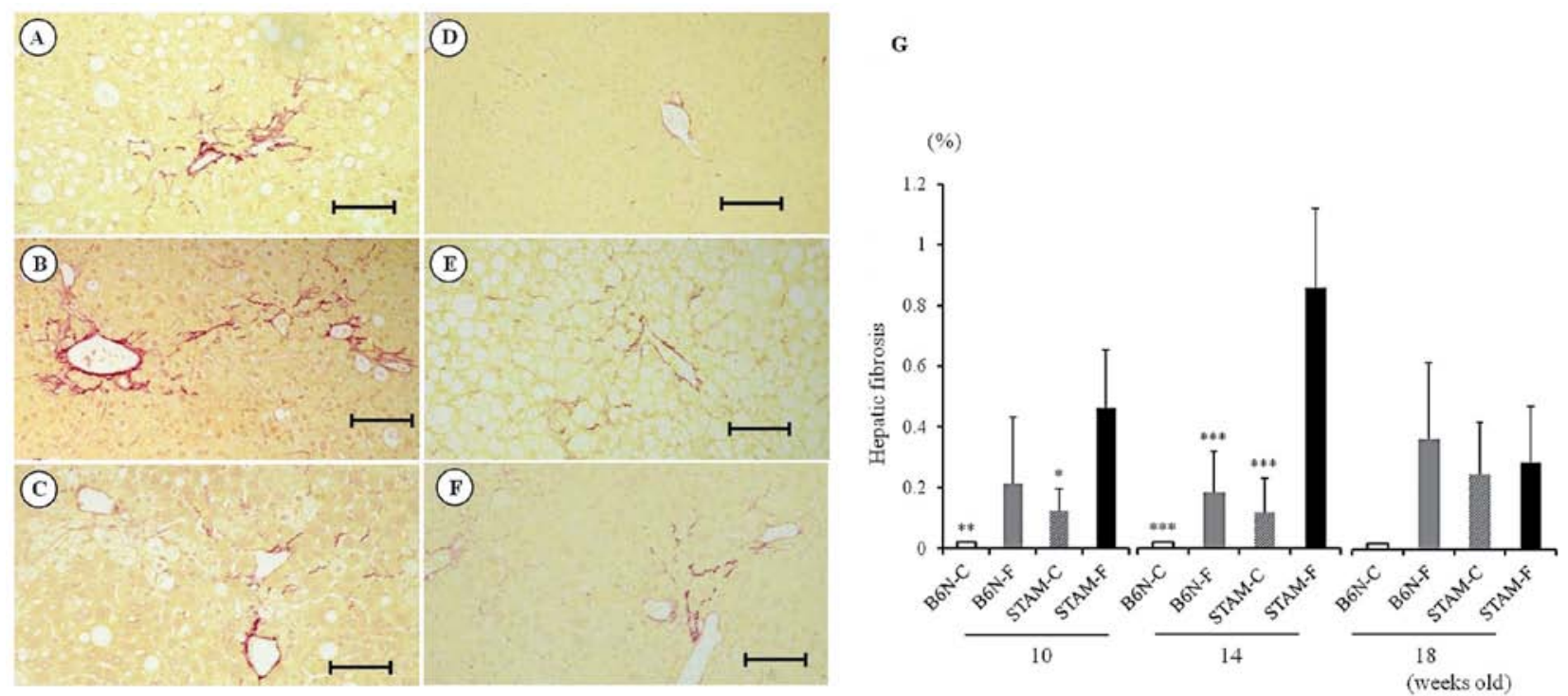

Figure 4. Hepatic fibrosis evaluated with Sirius red staining. (A-C) Sirius red staining of the liver in STAM-F mice at (A) 10 , (B) 14 and (C) 18 weeks. (D-F) Sirius red staining of the liver at 18 weeks in (D) B6N-C, (E) B6N-F, and (F) STAM-C groups. Original magnification, x100; scale bar, $100 \mu \mathrm{m}$. (G) Area positive for Sirius red staining in B6N and STAM mice at 10, 14 and 18 weeks ( $\mathrm{n}=5$ in each group). Statistical analyses were performed using Tukey's HSD test. " $\mathrm{p}<0.05,{ }^{* *} \mathrm{p}<0.01,{ }^{* * *} \mathrm{p}<0.001$ vs. each week's STAM-F.

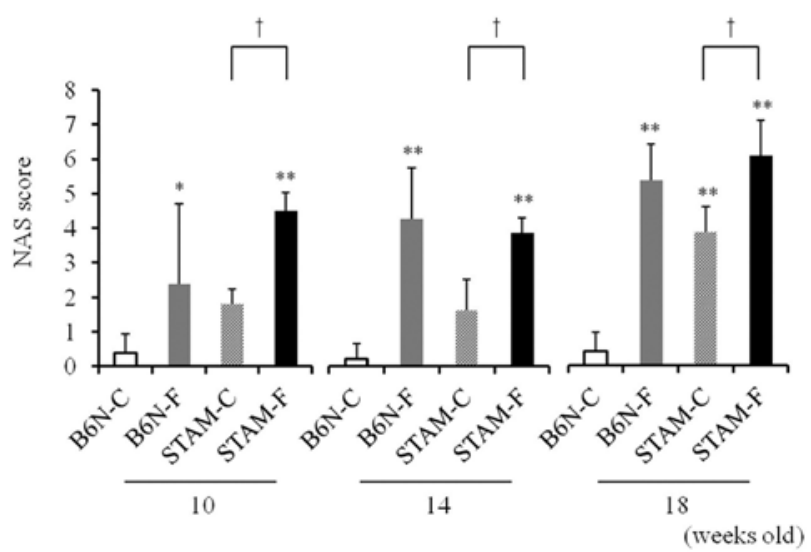

Figure 5. Assessment of NAFLD activity score (NAS). NAS of STAM-F and B6N-F groups was significantly higher than that of $\mathrm{B} 6 \mathrm{~N}-\mathrm{C}$ mice at 10,14 and 18 weeks. In addition, NAS of the STAM-F group was significantly higher than that of STAM-C mice at 10,14 and 18 weeks; $n=5$ per group. Statistical analyses were performed using Tukey's HSD test. "p $<0.05,{ }^{* *} \mathrm{p}<0.001$ vs. B6N-C in each week. ${ }^{\dagger} \mathrm{p}<0.01$, STAM-C vs. STAM-F. the STAM groups, the score was higher in STAM-F than in STAM-C groups (Fig. 5).

Hepatic tumor formation and evaluation of tumor in STAM mice. Hepatic tumors were observed in two STAM-C mice at 14 weeks of age. However, the tumors in each mouse were singular, and no hepatic tumors were observed in the STAM-C group at 10 and 18 weeks of age, or in either B6N group at any time during the experiment. By contrast, a single hepatic tumor was observed in all five STAM-F mice at 14 weeks, and multiple tumors were observed in all five STAM-F mice at 18 weeks (Fig. 6A).

Immunohistochemical analysis showed that hepatic tumors in STAM-F mice at 18 weeks were positive for GST-P (Fig. 6B) and HSP-70 (Fig. 6C). In addition, quantitative RT-PCR analysis of mRNA from liver tissue in STAM-F mice at 14 weeks revealed that expression of $A f p$ mRNA was significantly higher, and that of Gpc3 mRNA tended to be 

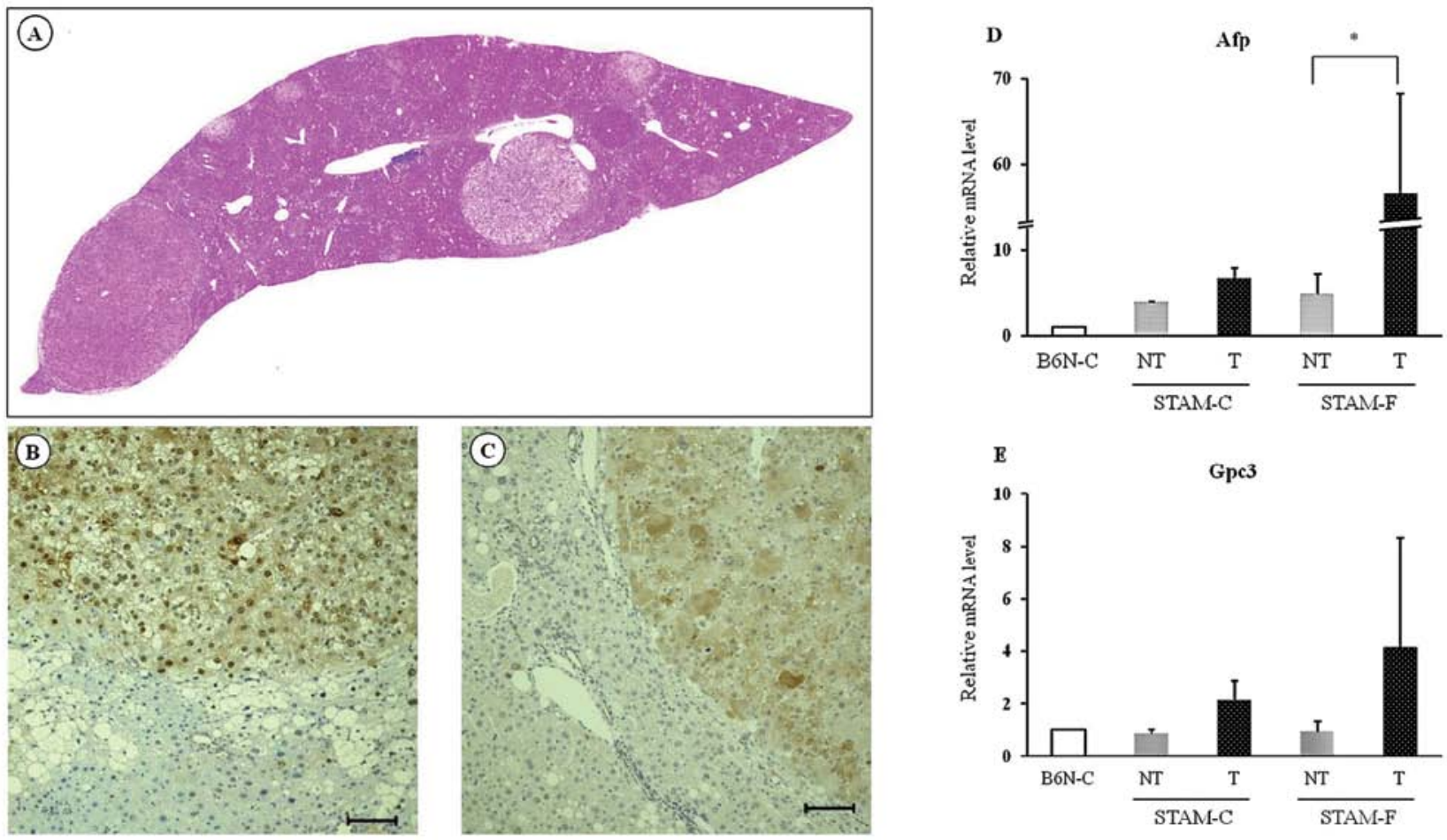

Figure 6. Evaluation of hepatic tumors in STAM-F mice at 14 or 18 weeks old. (A) Hematoxylin and eosin-stained sections are shown in STAM-F mice at 18 weeks old. There are multiple tumors. (B and C) Immunostaining for GST-P (B) and HSP-70 (C) in STAM-F mice at 18 weeks old. Original magnifications in B and C, x100; scale bar, $100 \mu \mathrm{m}$. (D and E) Assessment of mRNA expression of cancer-associated genes (D, Afp; E, Gpc3) in liver tissue by quantitative RT-PCR. Hepatic tumors found in liver tissues were obtained from 14-week-old STAM-F $(n=5)$ or STAM-C $(n=2)$ mice. NT, non-tumor; T, tumor. Statistical analyses were performed using the Mann-Whitney U test. " $\mathrm{p}<0.01, \mathrm{NT}$ vs. T.

higher, in the tumor area than in the non-tumor area (Fig. 6D and E). On this basis, we concluded that the hepatic tumors in STAM-F mice had features of hepatocellular carcinomas. By contrast, $A f p$ and $G p c 3$ mRNA expression in hepatic tumors of STAM-C mice at 14 weeks was similar to that in non-tumor areas (Fig. 6D and E).

cDNA microarray analysis and quantitative RT-PCR. Gene-expression profiles of whole livers from B6N-C, B6N-F, and STAM-F mice at 14 weeks, using en bloc total RNA from five mice, were evaluated using Affymetrix GeneChips. Of 2,774 genes whose expression levels were $\geq 2$-fold higher in STAM-F than B6N-F, mice 144 genes were increased $\geq 5$-fold, and 8 genes were increased $\geq 30$-fold. These top eight genes were selected for further examination (Table II). In addition, of 144 genes that were increased $\geq 5$-fold in the STAM-F compared to the B6N-F group, four genes were increased $\geq 5$-fold in the B6N-F compared to the B6N-C group at 14 weeks, and the four genes were selected for further examination (Table II). Of the 12 genes, expression levels of 11 were assessed by quantitative RT-PCR using commercially available primers. We confirmed that expression levels of the Sptlc3, Mmp13, Gripl and Cxcl14 genes were higher in the STAM-F as compared to the STAM-C and B6N-F groups at all times tested (Fig. 7). The difference in Sptlc3 mRNA expression was the most pronounced, and increased over time from 10 to 18 weeks (Fig. 7). By contrast, differences in the hepatic mRNA expression levels of Cxcl14 and $M m p 13$ at 18 weeks were not statistically significant among the four groups, although these genes were expressed at higher levels in the STAM-F group than in the other groups.
Table II. Results of microarray analysis.

\begin{tabular}{lccc}
\hline $\begin{array}{l}\text { Gene } \\
\text { ID }\end{array}$ & $\begin{array}{c}\text { Gene } \\
\text { symbol }\end{array}$ & $\begin{array}{c}\text { STAM-F/B6N-F } \\
\text { ratio }\end{array}$ & $\begin{array}{c}\text { B6N-F/B6N-C } \\
\text { ratio }\end{array}$ \\
\hline 12945 & Dmbt1 & 198 & 0.01 \\
74053 & Grip1 & 132 & 0.04 \\
17263 & Meg3 & 121 & 0.02 \\
59012 & Moxd1 & 114 & 0.84 \\
11717 & Ampd3 & 49 & 0.04 \\
102657 & Cd276 & 43 & 0.05 \\
20963 & Syk & 35 & 0.72 \\
57266 & Cxcl14 & 33 & 1.90 \\
17386 & Mmp13 & 18 & 7.0 \\
17381 & Mmp12 & 7.2 & 16 \\
192885 & F630007L15Rik & 6.6 & 5.7 \\
228677 & Sptlc3 & 5.9 & 5.7 \\
\hline
\end{tabular}

Comparison of gene expression between STAM, $d b / d b$ and CDAA mouse models. In $\mathrm{db} / \mathrm{db}$ mice fed HFD for 20 weeks (at 25 weeks old, db/db-F group), we observed steatosis and fibrosis (Fig. 8A and B) but no hepatic tumors. Similarly, hepatic steatosis and fibrosis, but no hepatic tumors, were observed in B6N mice fed a CDAA diet for 12 weeks (at 16 weeks old, CDAA group; Fig. 8C and D). The NAS of $\mathrm{db} / \mathrm{db}-\mathrm{F}$ and CDAA mice was as high as that of STAM-F mice (Fig. 8E), and hepatic fibrosis was significantly more severe in 

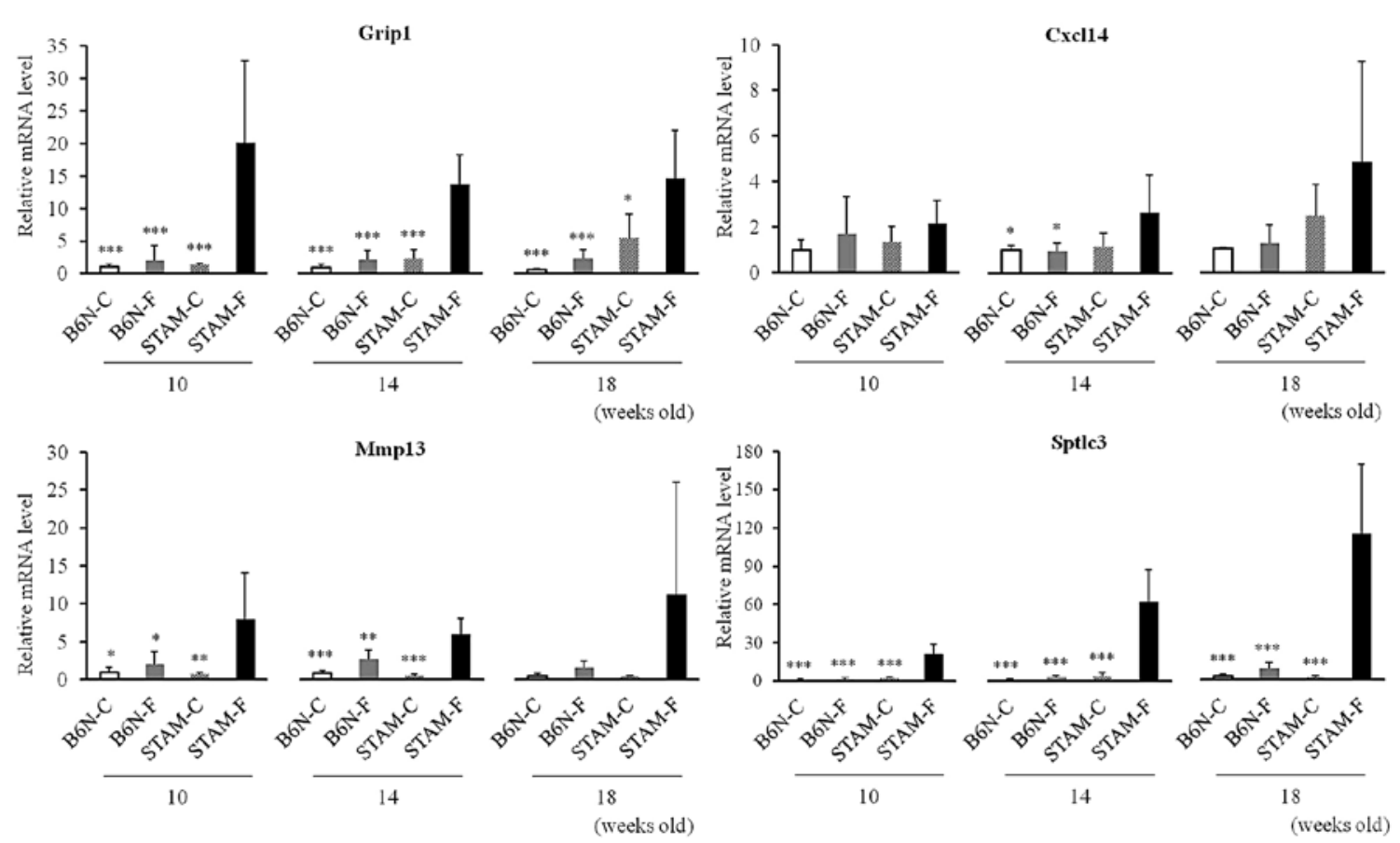

Figure 7. Hepatic mRNA expression levels assessed by quantitative RT-PCR.Data are shown as means \pm standard deviation ( $\mathrm{n}=5$ in each group). Statistical analyses were performed using Tukey's HSD test. ${ }^{*} \mathrm{p}<0.05,{ }^{* *} \mathrm{p}<0.01,{ }^{* * *} \mathrm{p}<0.001 \mathrm{vs}$. STAM-F in the same week.

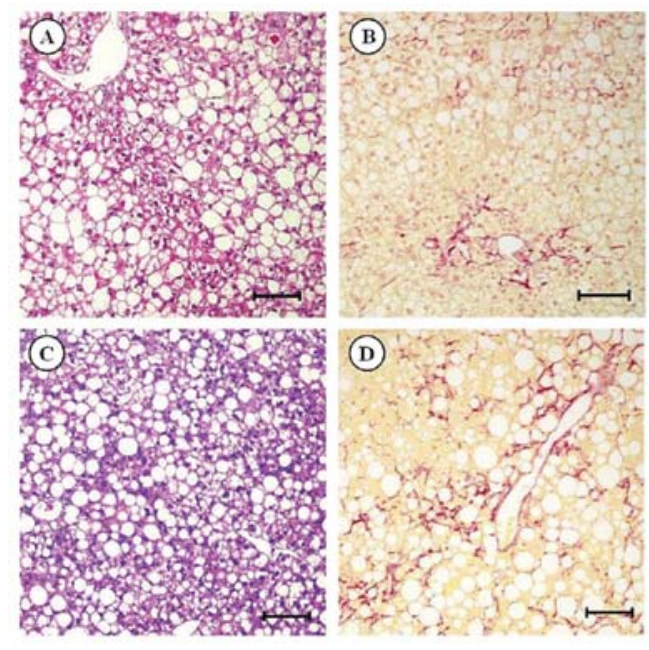

G

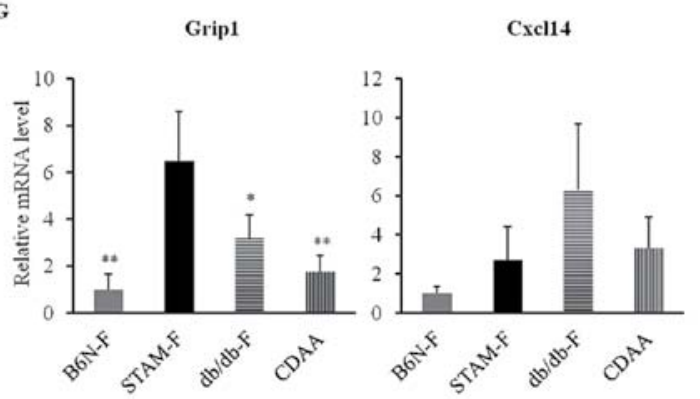

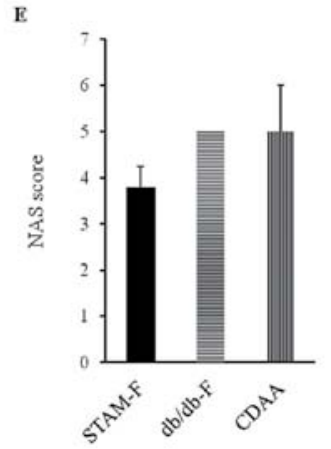

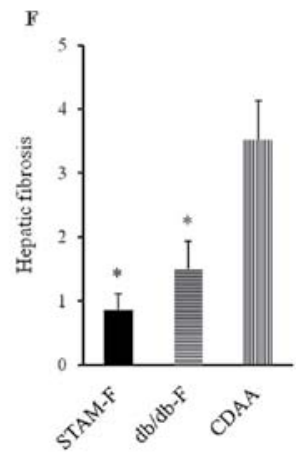

Mmp13

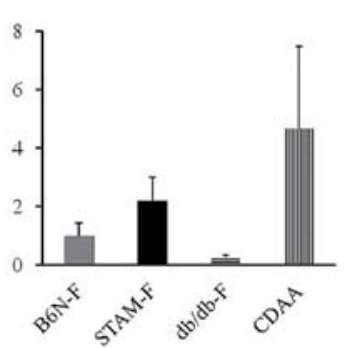

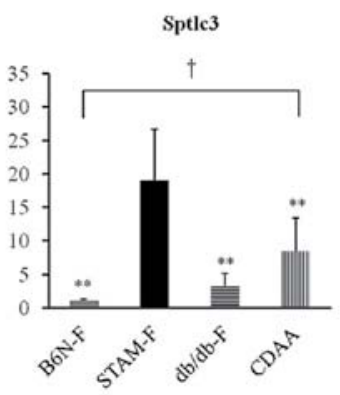

Figure 8. Liver histological findings and mRNA expression levels of liver tissue in the steatohepatitis mouse model. (A) Hepatic steatosis and (B) hepatic fibrosis are observed at 25 weeks in db/db mice fed a high-fat diet (db/db-F group), as determined by hematoxylin and eosin and Sirius red staining, respectively. (C) Hepatic steatosis and (D) hepatic fibrosis are shown in B6N mice fed a CDAA diet (CDAA group) at 16 weeks, assessed by hematoxylin and eosin and Sirius red staining, respectively. Original magnifications in A-D, x100; scale bar, $100 \mu \mathrm{m}$. (E) Assessment of NAS of STAM-F mice at 14 weeks, db/db-F mice at 25 weeks, and CDAA mice at 16 weeks ( $\mathrm{n}=5$ in each group). NAS, nonalcoholic fatty liver disease activity score. (F) Area positive for Sirius red staining in STAM-F mice at 14 weeks, db/db-F mice at 25 weeks, and CDAA mice at 16 weeks ( $n=5$ in each group). "p<0.001 vs. CDAA. (G) Hepatic mRNA expression levels of Gripl and Sptlc3 were highest in the STAM-F compared to the other three groups. By contrast, hepatic mRNA expression of Cxcl14 in the $\mathrm{db} / \mathrm{db}-\mathrm{F}$ group or Mmp13 in the CDAA group was relatively higher compared to the other three groups. Liver tissues were obtained from 14-week-old B6N-F or STAM-F, or CDAA mice at 16 weeks, or db/db-F mice at 25 weeks ( $\mathrm{n}=5$ in each group). Data are shown as means \pm standard deviation. Statistical analyses were performed using Tukey's HSD test. ${ }^{*} \mathrm{p}<0.01,{ }^{* *} \mathrm{p}<0.001$ vs. STAM-F; ${ }^{\dagger} \mathrm{p}<0.05$, B6N-F vs. CDAA. CDAA, choline-deficient L-amino acid-defined diet. 
the CDAA mice than in STAM-F mice at 14 weeks (Fig. 8F). Among these mice and the B6N-F and STAM-F groups, the hepatic expression of Gripl and Sptlc3 mRNAs was highest in the STAM-F group at 14 weeks (Fig. 8G). By contrast, the hepatic levels of $\mathrm{Cxcll4}$ and Mmpl3 mRNA were higher in the $\mathrm{db} / \mathrm{db}-\mathrm{F}$ and CDAA groups, respectively, as compared to the other groups (Fig. 8G).

\section{Discussion}

This study showed that the STAM-F group, which exhibited notable hyperglycemia due to impaired insulin secretion over a short time period, exhibited more hepatic fibrosis at 10 and 14 weeks and more frequent hepatocarcinogenesis at 18 weeks than the B6N-C, B6N-F and STAM-C groups. In addition, mRNA expression levels of several genes including Sptlc3 in the liver of STAM-F mice were significantly elevated compared to the STAM-C, B6N-C and B6N-F groups. Sptlc3 expression gradually increased over the study observation period. Furthermore, we observed a higher expression of Sptlc3 mRNA in STAM-F mice compared to NASH mouse models, such as db/db-F and CDAA diet mice, although hepatic fibrosis was more severe in the CDAA diet mice than in the STAM-F mice. These results indicate that a HFD in conjunction with hyperglycemia may aggravate pathophysiological findings in the liver, including hepatocarcinogenesis in NASH. Furthermore, hepatic expression of Sptlc3 mRNA may be closely associated with NASH-associated hepatocarcinogenesis.

Type 2 diabetes is a risk factor for HCC: the risk of HCC is 2.5-fold higher in diabetic patients than in healthy controls (12). Sixty-four percent of HCC patients suffering from NASH experience complications with diabetes (13). Several factors, such as hyperinsulinemia induced by insulin resistance, play major roles in hepatocarcinogenesis in NASH (14). By contrast, hyperglycemia itself can contribute to the incidence of carcinogenesis by promoting endothelial cell dysfunction or the induction of DNA damage by oxidative stress (15). In this study, multiple HCCs were observed in the STAM-F group, which had higher hyperglycemia than the STAM-C and B6N-F groups. Thus, our results show that significant hyperglycemia contributes to hepatocarcinogenesis, regardless of hypoinsulinemia.

HCCs express Afp and Gpc3 mRNA $(16,17)$, and immunostain positively for GST-P and HSP-70 of HCC $(18,19)$. These observations support the hypothesis that the liver tumors observed in STAM-F mice were HCCs. By contrast, no significantly higher expression of $A f p$ and $G p c 3$ mRNA was observed in hepatic tumors that were detected in two STAM-C mice at 14 weeks. In addition, the non-tumor area of the liver in these mice showed a low expression of Sptlc3. Therefore, Sptlc3 expression may contribute to the development of HCC with a higher expression of tumor markers such as AFP, but probably does not contribute to hepatic tumors that do not highly express these tumor markers.

In this study, HCC formation in STAM-F mice at 14 and 18 weeks was accompanied by a decrease in hepatic steatosis, which was similar to the features of burned-out NASH. However, STAM-F mice did not develop LC within the period when HCC was observed. Although LC is a risk factor for
HCC development, HCC in patients with metabolic syndrome often develops without advanced hepatic fibrosis (20). Thus, development of HCC in STAM-F mice with mild fibrosis may be similar to that in human NASH, although the mechanism of the decrease in hepatic steatosis in STAM-F has not been elucidated.

In the STAM group, we observed fasting hyperglycemia after the impairment of pancreatic function. In addition, blood glucose levels gradually decreased in STAM mice fed a conventional diet (STAM-C), but gradually increased in STAM mice fed a high-fat diet (STAM-F). The palmitic acid from the high-fat diet may inactivate AMP-activated protein kinase, increase production of reactive oxygen species (ROS) in mitochondria, and decrease autophagic signaling via Unc-51-like kinase 1 (ULK1). These factors promote the production of IL-1 $\beta$, impair glucose tolerance and insulin sensitivity (21), and may lead to hepatocarcinogenesis. Oxidative stress such as ROS production should be further investigated in STAM mice.

Serine palmitoyltransferase (SPT) produces 3-ketodihydrosphingosine (KDS) by condensation reactions with serine and palmitoyl CoA using pyridoxal 5'-phosphate (PLP) as a coenzyme $(22,23)$. This response is the first step in the biosynthetic pathway of sphingolipids. Although the mammalian SPT was previously described as a heterodimer composed of two subunits, SPTLC1 and SPTLC2 (24), a recent study has shown that SPT is composed of three distinct subunits (SPTLC1, SPTLC2 and SPTLC3) that form a complex with a molecular mass of $480 \mathrm{kDa}(25)$. SPTLC2 and SPTLC3 share $68 \%$ sequence identity and include the PLP-binding region, whereas SPTLC1 does not contain that region (24). Therefore, SPTLC2 and SPTLC3 are likely to have similar functions. However, SPTLC2 and SPTLC3 are encoded by two distinct genes, and Triton X-100 inhibits the SPTLC3-based SPT reaction but activates the SPTLC2-mediated reaction (24). Our results indicated that HCC development was associated with mRNA expression of Sptlc3, but not Sptlc1 or Sptlc2 (data not shown). Differences in the functions of these genes may be associated with hepatocarcinogenesis.

The levels of sphingolipids such as ceramide are correlated with the expression level of Sptlc3 (26). Blockade of SPT1, an enzyme involved in the rate-determining step of ceramide synthesis, improves insulin resistance in mice (27). The expression of pro-ceramide genes such as Sptlcl and Sptlc2 is increased in the early stage of fatty liver disease in mice, and serum ceramide levels of NASH patients decrease in accordance with their weight reduction (27-29). Inhibition of ceramide de novo synthesis with the SPT inhibitor myriocin ameliorates glucose homeostasis in streptozotocin-induced type 1 diabetes in rat (30). Thus, ceramide may be associated with fatty liver in a manner that involves insulin resistance. In addition, myriocin suppressed melanoma cell proliferation by cell-cycle arrest at the $\mathrm{G} 2 / \mathrm{M}$ phase by inhibiting de novo sphingolipid synthesis and increased p53 and p21 ${ }^{\text {wafl/cipl }}$ expression $(31,32)$. In this study, overexpression of Sptlc3 was already detected at 10 weeks in STAM-F, prior to liver tumor formation. Furthermore, expression of Sptlc3 increased in accordance with progression to HCC. Therefore, sphingolipids controlled by Sptlc3 might be associated with hepatocarcinogenesis via mechanisms involving insulin resistance, cell proliferation, and p53/p21 waf1/cipl expression. 
Our study had several limitations. First, the STAM mice that were used in this study developed NASH in the context of hyperglycemia and impaired insulin secretion. The pathological condition in this model is different from that of human NASH, in which hyperinsulinemia is associated with obesity and insulin resistance. However, previous studies have reported NAFLD cases being on the increase due to type 1 diabetes, and described the influence of insulin resistance on the development of NAFLD in such patients (33-35). Although several mouse models of NASH exist, the development of HCC has not been observed in these models. Therefore, STAM-F are considered to be useful as a highly reproducible NASH model. A second limitation is that STAM-F mice develop hepatic steatosis and hepatic fibrosis, followed by HCC, but the area of hepatic fibrosis (as determined by image analyzer in STAM-F at 18 weeks) was reduced relative to the area at 10 or 14 weeks. Although this reduction is inconsistent with findings in human NASH, it is necessary to assess hepatic fibrosis in the tumor-free area in many mice. Third, it is uncertain whether several genes that our study found to be increased in STAM-F are specific to this mouse model or are actually increased in human NASH associated with insulin resistance and hyperinsulinemia. Fourth, our study did not conclusively demonstrate an actual role of Sptlc3 in the development of NASH and NASH-associated tumor formation. Direct evidence that Sptlc3 is involved in the progression to NASH would require experimental evidence that the suppression of Sptlc3 ameliorates NASH or the overexpression of Sptlc3 exacerbates NASH. Finally, there was no apparent hepatic steatosis in STAM-C or B6N-C mice. The livers of STAM-C and STAM-F mice may show inflammation caused by streptozotocin hepatotoxicity, which could affect the NAS score. Thus, we should consider that there are limitations to the NAS score used for evaluation in our model.

In summary, we found that a high-fat diet, marked hyperglycemia, and hepatic steatosis were correlated with multiple HCC, and concluded that these factors contributed to the progression of NASH. In addition, overexpression of Sptlc3 in the liver was associated with NASH-associated hepatocarcinogenesis. This study provides useful information regarding potential molecular targets for the prevention and treatment of NASH-associated hepatocarcinogenesis, although the pathology of STAM is somewhat different from that of human NASH, in particular with regard to insulin resistance.

\section{Acknowledgements}

We would like to thank Ms. Yuko Morinaga as well as Ms. Etsuko Horiguchi for their technical assistance.

\section{References}

1. Ludwig J, Viggiano TR, McGill DB and Oh BJ: Nonalcoholic steatohepatitis: Mayo Clinic experiences with a hitherto unnamed disease. Mayo Clin Proc 55: 434-438, 1980.

2. Andy SY and Keeffe EB: Nonalcoholic fatty liver disease. Rev Gastrointest Disord 2: 11-19, 2002.

3. Angulo P and Lindor KD: Non-alcoholic fatty liver disease Quadrennial review. J Gastroenterol Hepatol 17: S186-S190, 2002 .
4. White DL, Kanwal F and El-Serag HB: Association between nonalcoholic fatty liver disease and risk for hepatocellular cancer, based on systematic review. Clin Gastroenterol Hepatol 10: 1342-1359, 2012.

5. IkaiI,Arii S,OkazakiM,Okita K,OmataM,Kojiro M,Takayasu K, Nakanuma Y, Makuuchi M, Matsuyama Y, Monden M and Kudo M: Report of the 17th Nationwide follow-up survey of primary liver cancer in Japan. Hepatol Res 37: 676-691, 2007.

6. Day CP and James OF: Steatohepatitis: a tale of two 'hits'? Gastroenterology 114: 842-845, 1998.

7. Tilg $\mathrm{H}$ and Moschen AR: Evolution of inflammation in nonalcoholic fatty liver disease: the multiple parallel hits hypothesis. Hepatology 52: 1836-1846, 2010.

8. Anstee QM and Goldin RD: Mouse models in non-alcoholic fatty liver disease and steatohepatitis research. Int J Exp Pathol 87: $1-16,2006$.

9. Denda A, Kitayama W, Kishida H, Murata N, Tsutsumi M, Tsujiuchi T, Nakae D and Konishi Y: Development of hepatocellular adenomas and carcinomas associated with fibrosis in C57BL/6J male mice given a choline-deficient, L-amino aciddefined diet. Jpn J Cancer Res 93: 125-132, 2002.

10. Fujii M, Shibazaki Y, Wakamatsu K, Honda Y, Kawauchi Y, Suzuki K, Arumugam S, Watanabe K, Ichida T, Asakura H and Yoneyama H: A murine model for non-alcoholic steatohepatitis showing evidence of association between diabetes and hepatocellular carcinoma. Med Mol Morphol 46: 141-152, 2013.

11. Kleiner DE, Brunt EM, Van Natta M, Behling C, Contos MJ, Cummings OW, Ferrell LD, Liu YC, Torbenson MS, UnalpArida A, Yeh M, Mccullough AJ, Sanyal AJ and Nonalcoholic Steatohepatitis Clinical Research N: Design and validation of a histological scoring system for nonalcoholic fatty liver disease. Hepatology 41: 1313-1321, 2005.

12. Renehan A, Smith U and Kirkman MS: Linking diabetes and cancer: a consensus on complexity. Lancet 375: 2201-2202, 2010.

13. Rosmorduc O and Fartoux L: HCC and NASH: how strong is the clinical demonstration? Clin Res Hepatol Gastroenterol 36: 202-208, 2012.

14. Shimizu M,Tanaka T and MoriwakiH: Obesity and hepatocellular carcinoma: targeting obesity-related inflammation for chemoprevention of liver carcinogenesis. Semin Immunopathol 35: 191-202, 2013.

15. Morss AS and Edelman ER: Glucose modulates basement membrane fibroblast growth factor-2 via alterations in endothelial cell permeability. J Biol Chem 282: 14635-14644, 2007.

16. Niwa Y, Matsumura M, Shiratori Y, Imamura M, Kato N, Shiina S, Okudaira T, Ikeda Y, Inoue T and Omata M: Quantitation of alpha-fetoprotein and albumin messenger RNA in human hepatocellular carcinoma. Hepatology 23: 1384-1392, 1996.

17. Yao M, Yao DF, Bian YZ, Wu W, Yan XD, Yu DD, Qiu LW, Yang JL, Zhang HJ, Sai WL and Chen J: Values of circulating GPC-3 mRNA and alpha-fetoprotein in detecting patients with hepatocellular carcinoma. Hepatobiliary Pancreat Dis Int 12: 171-179, 2013.

18. Satoh K, Takahashi G, Miura T, Hayakari M and Hatayama I: Enzymatic detection of precursor cell populations of preneoplastic foci positive for gamma-glutamyltranspeptidase in rat liver. Int J Cancer 115: 711-716, 2005.

19. Shin E, Ryu HS, Kim SH, Jung H, Jang JJ and Lee K: The clinicopathological significance of heat shock protein 70 and glutamine synthetase expression in hepatocellular carcinoma. J Hepatobiliary Pancreat Sci 18: 544-550, 2011.

20. Paradis V, Zalinski S, Chelbi E, Guedj N, Degos F, Vilgrain $\mathrm{V}$, Bedossa P and Belghiti J: Hepatocellular carcinomas in patients with metabolic syndrome often develop without significant liver fibrosis: a pathological analysis. Hepatology 49: 851-859, 2009.

21. Wen H, Gris D, Lei Y, Jha S, Zhang L, Huang MT, Brickey WJ and Ting JP: Fatty acid-induced NLRP3-ASC inflammasome activation interferes with insulin signaling. Nat Immunol 12: 408-415, 2011.

22. Hanada K: Serine palmitoyltransferase, a key enzyme of sphingolipid metabolism. Biochim Biophys Acta 1632: 16-30, 2003.

23. Menaldino DS, Bushnev A, Sun A, Liotta DC, Symolon H, Desai K, Dillehay DL, Peng Q, Wang E, Allegood J, Trotman-Pruett S, Sullards MC and Merrill AH Jr: Sphingoid bases and de novo ceramide synthesis: enzymes involved, pharmacology and mechanisms of action. Pharmacol Res 47: 373-381, 2003. 
24. Hornemann T, Richard S, Rütti MF, Wei Y and Von Eckardstein A: Cloning and initial characterization of a new subunit for mammalian serine-palmitoyltransferase. J Biol Chem 281: 37275-37281, 2006

25. Hornemann T, Wei Y and Von Eckardstein A: Is the mammalian serine palmitoyltransferase a high-molecular-mass complex? Biochem J 405: 157-164, 2007.

26. Cinar R, Godlewski G, Liu J, Tam J, Jourdan T, Mukhopadhya B, Harvey-White $\mathbf{J}$ and Kunos G: Hepatic cannabinoid-1 receptors mediate diet-induced insulin resistance by increasing de novo synthesis of long-chain ceramides. Hepatology 59: 143-153, 2014.

27. Ussher JR, Koves TR, Cadete VJ, Zhang L, Jaswal JS, Swyrd SJ, Lopaschuk DG, Proctor SD, Keung W, Muoio DM and Lopaschuk GD: Inhibition of de novo ceramide synthesis reverses diet-induced insulin resistance and enhances whole-body oxygen consumption. Diabetes 59: 2453-2464, 2010.

28. Lyn-Cook LE Jr, Lawton M, Tong M, Silbermann E, Longato L, Jiao P, Mark P, Wands JR, Xu H and de La Monte SM: Hepatic ceramide may mediate brain insulin resistance and neurodegeneration in type 2 diabetes and non-alcoholic steatohepatitis. J Alzheimers Dis 16: 715-729, 2009.

29. Promrat K, Longato L, Wands JR and De La Monte SM: Weight loss amelioration of non-alcoholic steatohepatitis linked to shifts in hepatic ceramide expression and serum ceramide levels. Hepatol Res 41: 754-762, 2011.
30. Kurek K, Wiesiolek-Kurek P, Piotrowska DM, Lukaszuk B, Chabowski A and Zendzianendzian-Piotrowska M: Inhibition of ceramide de novo synthesis with myriocin affects lipid metabolism in the liver of rats with streptozotocin-induced type 1 diabetes. Biomed Res Int 2014: 980815, 2014.

31. Lee YS, Choi KM, Choi MH, Ji SY, Lee S, Sin DM, Oh KW, Lee YM, Hong JT, Yun YP and Yoo HS: Serine palmitoyltransferase inhibitor myriocin induces growth inhibition of B16F10 melanoma cells through $\mathrm{G}(2) / \mathrm{M}$ phase arrest. Cell Prolif 44: 320-329, 2011.

32. Lee YS, Choi KM, Lee S, Sin DM, Lim Y, Lee YM, Hong JT, Yun YP and Yoo HS: Myriocin, a serine palmitoyltransferase inhibitor, suppresses tumor growth in a murine melanoma model by inhibiting de novo sphingolipid synthesis. Cancer Biol Ther 13: 92-100, 2012.

33. Greenbaum CJ: Insulin resistance in type 1 diabetes. Diab Metab Res Rev 18: 192-200, 2002.

34. Bulum T, Kolaric B, Duvnjak L and Duvnjak M: Nonalcoholic fatty liver disease markers are associated with insulin resistance in type 1 diabetes. Dig Dis Sci 56: 3655-3663, 2011.

35. Regnell SE and Lernmark A: Hepatic steatosis in type 1 diabetes. Rev Diab Stud 8: 454-467, 2011. 